\title{
Alloy scattering in quantum well wire structures of semiconductor ternaries
}

\author{
G.B. Ibragimov \\ Institute of Physics, Azerbaijan National Academy of Sciences, 33 Javid av., 370143 Baku, Azerbaijan \\ E-mail: guseyn@physics.ab.az,guseyn_gb@mail.ru
}

\begin{abstract}
We studied the effect of the alloy-disorder-scattering on the electron transport in a quasi-one-dimensional semiconductor. Performed were analytical calculations of the alloydisorder-limited momentum relaxation time for carrier scattering in a cylindrical quantum wire using modeling wave functions, when the transverse part of the carrier wave function is taken as a Bessel function. It is found that the one-dimensional mobility is significantly greater than two-dimensional one. It is shown that the alloy-disorder-scattering-limited mobility increases with the increasing wire radius and increases with the increasing temperature. We compare our results with different scattering mechanisms for one-dimensional systems. Results are also included for the alloy composition dependence of the mobility.
\end{abstract}

Keywords: electron scattering, quantum well, quantum wire.

Paper received 10.07.02; accepted for publication 17.12.02.

\section{Introduction}

Recently there has been growing interest in the study of properties of ultrathin semiconducting wires, also called as quantum well wires with submicron dimensions. Electrons in a semiconductor quantum wire can be considered as a quasi one-dimensional (Q1D) electron gas [15]. There are Q1D structures in which the carriers are not confined to move along the length of the wire and the motion is quantized in the transverse directions. The physical properties of low-dimensional semiconducting structures such as electron transport characteristics differ from those of bulk semiconductors, because the translational symmetry is broken [6]. Sakaki [1] suggested using semiconductor quantum wires as a basis for very fast transport processes. Reduction of phase space in Q1D leads to a decrease in the scattering process, therefore, the mobility of the carriers has to increase compared to the bulk value. These high mobilities could be utilized in high-speed device applications [1,7]. The mobility of the electrons in the Q1D electron gas is limited by various scattering processes, which have relative importance at different ranges of temperature and carrier concentration. There have been calculations of electron scattering by acoustic phonon [8], impurity-limited mobility [2], phonon-limited mobility [3], the mobility of electrons scattered by impurities, by acoustic and polar optical phonons [4,9]. Alloy-disorder scattering is an important scattering mechanism when the confining quantum wire consists of a ternary semiconductor. Alloy-disorder scattering in ternary compound semiconductors and quantum well structures has been subject of many theoretical and experimental investigations [10-24]. In its conventional theory, the constituent type $\mathrm{A}$ and type $\mathrm{B}$ atom pairs are assumed to be distributed randomly within the volume of the crystal.

The main purpose of this paper is to derive approximate analytical expressions for the momentum relaxation time associated with the electron-alloy-disorder interactions, and the calculation of the mobility of Q1D electron gas. The outline of this paper is as follows. In Sec.II we give the general framework of the paper. In Sec.III we present the calculation of the momentum relaxation time for alloydisorder scattering. In Sec.IV the quantum theory of the alloy-disorder limited mobility of thin semiconduting wires is presented. In Sec.V some numerical results of our calculation are presented for $\operatorname{In}_{1-x} \mathrm{Ga}_{\mathrm{x}}$ As. Finally, a brief discussion about our numerical analysis is given. 


\section{G.B. Ibragimov: Alloy scattering in quantum well wire structures ...}

\section{General Framework}

We assume that the electrons are confined to move in a cylindrical wire of radius $R$ and are free to move along the axis of the wire, which is of length $L$. Within the framework of effective-mass approximation the electron wave function in quantum wire is given by [25]

$$
\Psi_{n l k(r)}=\frac{\exp (i K z) \exp (i l \vartheta)}{\left(\pi R^{2} L\right)^{1 / 2}} \varphi_{n l}(\rho)
$$

where $l=0,1,2,3, \ldots ; n=1,2,3, \ldots$;

$$
\varphi_{n l}(\rho)=\frac{J_{l}\left(k_{n l} \rho\right)}{J_{l+1}\left(k_{n l} R\right)}
$$

Here $r$ is $(\rho, \vartheta, z), \vartheta$ is the azimuthal angle about the wires, $K$ is the wave vector of the electron along the $z$ axis, which is chosen along the axis of the cylindrical wire, $J_{l}(x)$ is the Bessel function of the first kind with order $l$. In order to satisfy the boundary condition that $\Psi(\rho=R)=0, k_{n l}$ is the $n$-th zero of $J_{l}\left(k_{n l} R\right)$.

The eigenvalues corresponding to Eq.(1) are

$$
E_{n l k}=E_{k}+\frac{\hbar^{2} k_{n l}^{2}}{2 m^{*}}
$$

where $E_{k}$ is the electron kinetic energy along the $z$ direction, and $m^{*}$ is the electron effective mass. When the de Brogile wavelength of electrons becomes comparable to the transverse dimensions of the wire, the electrons are confined to their lowest guantum state as far as their transverse motion is concerned ( $n=1, l=0$ for a cylindrical wire) while their motion along the axis of the wire is unaffected [26]. For such a ground state wave function $k_{10}=2.403 / R$.

When the confining quantum well consists of a ternary semiconductor (like $\mathrm{Ga}_{1-\mathrm{x}} \mathrm{In}_{\mathrm{x}} \mathrm{As}$ ), in the virtual crystal approximation alloy-disorder scattering potential has the form [17-20]:

$$
H_{d i s}=\delta V\left\{(1-x) \sum_{r_{I n}} Y_{\Omega_{0}}\left(r-r_{I n}\right)-x \sum_{r_{G a}} Y_{\Omega_{0}}\left(r-r_{G a}\right)\right\}, \text { (4) }
$$

where $Y_{\Omega_{0}}\left(r_{a}-r_{b}\right)=1 / \Omega_{0}$ when $r_{a}$ and $r_{b}$ are inside the same unity cell and vanishes elsewhere, and the summations run over all the unit cells, $\Omega_{0}$ is the volume of the unit cell.

The momentum relaxation time $\tau$ of the electrons in a Q1D system due to the scattering potential $H_{d i s}$ is given by the relaxation rate [27]

$$
\tau^{-1}=\frac{2 \pi}{\hbar} \sum_{f}|<f| H_{d i s}|i>|^{2}(1-\cos \theta) \delta\left(E_{f}-E_{i}\right)
$$

Here, $i$ and $f$ represent the initial and final states and $\mathrm{q}$ is the angle between the incident and scattered wave vectors of electrons along the axis of the wire. Because of the Q1D nature of the electron gas in a thin wire in the quantum limit, the scattering angle $\theta$ is limited to two values 0 and p. Thus, the value $(1-\cos \theta)$ in Eq. (5) will be 2 . The total wave function of the state " $f$ " is $\Psi_{f T}=\Psi_{f} u_{c}\left(r_{e}\right)$, where $u_{c}\left(r_{e}\right)$, are Bloch functions corresponding to the extrema of the conduction band.

In order to evalute the matrix elements $\left\langle f\left|H_{d i s}\right| i>\right.$ in Eq. (5), we replace $r_{e} \rightarrow r_{e}+r_{I n(G a)}$ and use the relations: $u_{c}\left(r_{e}+r_{I n(G a)}\right)=u_{c}\left(r_{e}\right)$ and $f_{\Omega_{0}} d^{3} r\left|u_{c}(r)\right|^{2}=\Omega_{0}$. The scattering rate induced by the alloy-disorder is calculated using the statistical average of products of two different matrix elements.

The mobility of electrons confined to Q2D quantum well and free to move along the $Z$ axis of the thin semiconducting wire is given in the relaxation time approximation by

$$
\mu=e\left[\sum_{K}\left(\frac{\hbar K}{m^{*}}\right) \tau\left(E_{K}\right) \frac{\partial f_{0}\left(E_{K}\right)}{\partial E_{K}}\right]\left(\sum f_{0}\left(E_{K}\right)\right)^{-1}
$$

where $f_{0}\left(E_{K}\right)$ is the electron distribution function for the carriers in the wire.

\section{Momentum scattering rate for alloy disorder scattering}

Using the wave functions given by Eqs.(1) and (3), the matrix elements induced by the alloy-disorder become

$$
\begin{aligned}
& <f\left|H_{d i s}\right| i>= \\
& =\frac{\delta V}{\pi R^{2} L} \sum_{r_{p}} \varphi_{10}^{2}(\rho) e^{i \Delta K Z_{p}\left[(1-x) \delta_{p, I n}-x \delta_{p, G a}\right]}
\end{aligned}
$$

where $\Delta K=K-K^{\prime}$. The summation on $r_{p}$ is over all the crystal unit cells and $\delta_{p, I n}=1(=0)$ if the cell at $r_{p}$ contains one In (respectively, Ga) atom.

A straightforward calculation of the square of the matrix element in (7) gives

$k f\left|H_{d i s}\right| i>\left.\right|^{2}=\frac{\Omega_{0}(\delta V)^{2} x(1-x)}{\pi R^{4} L} G(R)$

where

$$
G(R)=\int_{0}^{R} \rho \varphi_{10}^{4}(\rho) d \rho
$$




\section{G.B. Ibragimov: Alloy scattering in quantum well wire structures ...}

When (5) and (8) are combined, the momentum relaxation rate for electron-alloy-disorder scattering is obtained:

$$
\frac{1}{\tau}=\frac{2 \Omega_{0}(\delta V)^{2} x(1-x) m^{*}}{\pi \hbar^{3} R^{4} K} G(R)
$$

which combined with $E_{K}=\hbar^{2} K^{2} / 2 m *$ gives $\tau\left(E_{K}\right)$ explicity as a function of the energy.

Since we are interested in the ground state approximation, we may employ the approximate radial wave function proposed in [28]

$$
\varphi_{10} \approx \sqrt{3}\left[1-\frac{\rho^{2}}{R^{2}}\right]
$$

which gives the corresponding $G(R)=\frac{9 R^{2}}{12}$. At this point, the momentum relaxation rate for electron-alloy-disorder scattering changes into

$$
\tau^{-1}=\frac{3 \Omega_{0}(\delta V)^{2} x(1-x) m^{*}}{2 \pi \hbar^{3} R^{2} K} .
$$

Eqs. (9)-(10) shows that the scattering rate increases and the momentum relaxation time decreases as the radius of the wire decreases due to scattering caused by the alloy-disorder.

A formula similar to Eq. (10) has been obtained by Ando [19] and Bastard [18] for a Q2D electron gas.

\section{Mobility}

In the general case, where there are no approximations for $f_{0}(E)$ using Eq. (9) in Eq. (6) the mobility can be written as

$$
\mu=\frac{2 e \hbar R^{4} K_{b} T F_{1}(\eta)}{m * \Omega_{0}(\delta V)^{2} x(1-x) n_{1 D} G(R)}
$$

Here $n_{1 D}=2 \sqrt{2 K_{B} T m *} F_{1 / 2}(\eta) / \pi \hbar$ is the density of electrons per unit length of the wire, $F_{n}(\eta)$ is the Fermi integral of the argument $\eta=\varsigma / K T$ [27], and $\varsigma$ is the chemical potential. It can be seen that in Eq. (11) the mobility increases as the wire radius increases.

For the case of non-degenerate carrier statistics, $f_{0}(E)$ is given by the Maxwell-Boltzmann distribution and mobility is given by

$$
\mu_{n o n . \mathrm{deg} .}=\frac{\pi^{1 / 2} e \hbar^{2} R^{4}\left(K_{B} T\right)^{1 / 2}}{\sqrt{2} m^{* 3 / 2} \Omega_{0}(\delta V)^{2} x(1-x) G(R)}
$$

When the carriers are degenerate, at low temperature, $f_{0}(E)$ is given by the Fermi-Dirac distribution and mobility is $e \tau\left(E_{F}\right) / m^{*}$ :

$\mu_{\text {deg. }}=\frac{\pi e \hbar^{3} R^{4} K_{B}}{2 m^{2} \Omega_{0}(\delta V)^{2} x(1-x) G(R)}$

where $E_{F}$, the Fermi energy of electrons in a degenerate $1 \mathrm{D}$ electron gas, is $\hbar^{2} K_{F}^{2} / 2 m *$ with $K_{F}=\pi n_{1 D} / 2$.

It is noteworthy that the mobility due to alloy-disorder scattering in 3- and 2-dimensional systems has a functional dependence on the temperature according to $T^{-1 / 2}$ [10] and $T^{0}[13,21]$, respectively.

Eqs (12)-(13) show that the $m$ varies as $T^{0}$ at low temperatures and as $\mathrm{T}^{1 / 2}$ at high temperatures. This occurs because the density of states in a 3-, 2-, and 1-dimensional system has a functional dependence on energy according to $E^{1 / 2}, E^{0}$ and $E^{-1 / 2}$, respectively.

If $\mu_{0}$ is the alloy-scattering mobility in the Q2D system[22], then for a nondegenrate electron distribution

$\left(\frac{\mu}{\mu_{0}}\right)_{\text {non.deg }}=\frac{3.48 R^{2}\left(K_{B} T m^{*}\right)^{1 / 2}}{d \hbar}$

where $d$ is the thickness of the well. For $\mathrm{Ga}_{0.47} \operatorname{In}_{0.53} \mathrm{As}$ with $d=R=10^{-6} \mathrm{~cm}$, we obtain $\left(\mu / \mu_{0}\right)_{\text {non.deg }}=0.247 T^{S}$, so that the alloy-scattering mobility in the quantum wire is higher than that in the Q2D systems for temperatures above $17 \mathrm{~K}$.

On the other hand, for a completely degenerate electron distribution we have

$$
\left(\frac{\mu}{\mu_{0}}\right)_{\mathrm{deg} .}=\frac{6.14 R^{2}\left(m^{*} E_{F}\right)^{1 / 2}}{d \hbar}
$$

For $\mathrm{G} a_{0.47} \operatorname{In} 0{ }_{53}$ As with $d=R=10^{-6} \mathrm{~cm}$ and $E_{F}=$ $=4 K_{B} T$ we find $\left(\mu / \mu_{0}\right)_{\text {deg. }}=0.86 T^{1 / 2}$, so that quantum wire mobility is higher than that in the Q2D systems for temperatures above $1 \mathrm{~K}$.

\section{Numerical analysis and discussion}

In this paper, the alloy-disorder-scattering limited momentum relaxation rate and mobility for carriers confined to a quantum well wire has been determined. As a numerical example, we consider $\mathrm{Ga}_{\mathrm{x}} \mathrm{In}_{1-\mathrm{x}}$ As quantum wire. Certain material parameters such as the effective mass $m^{*}$ and lattice parameter occur in various formulae for mobility (relaxation time).

The momentum relaxation rate, $\tau^{-1}$ as a function of the longitudinal energy $E_{K}$ in the $\mathrm{Ga}_{0.8} \mathrm{In}_{0.2} \mathrm{As}$ is shown in Fig. 1. This numerical results are plotted from Eq. (9) 


\section{G.B. Ibragimov: Alloy scattering in quantum well wire structures ...}

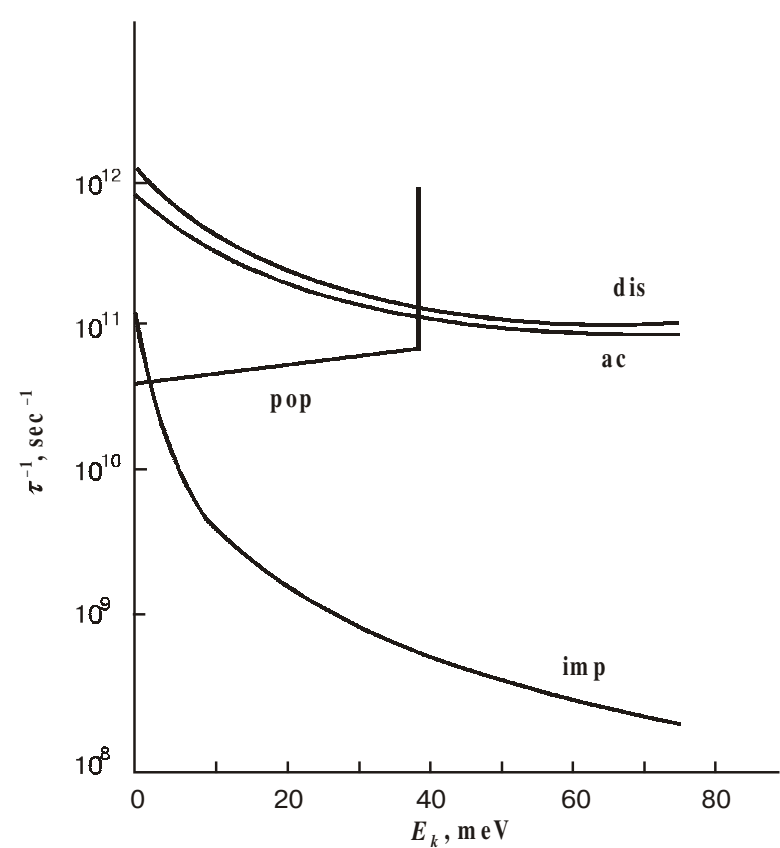

Fig. 1. For a $1 \mathrm{D} \mathrm{Ga}_{0.2} \operatorname{In}_{0.8}$ As system, the momentum relaxation rate is plotted as function of the electron energy $E_{K}$ for alloydisorder scattering. Scattering rates for scattering from background impurity, acoustic phonon and polar optic phonon are shown for 1D GaAs system [4].

for scattering from alloy-disorders. In order to compare the momentum relaxation rate for the alloy-disorder scattering with momentum relaxation rate for various scattering mechanisms, $\tau^{-1}$ values for GaAs thin wire structures found in the literature [4] and are plotted in Fig.1. It is shown that for impurity, alloy-disorder and acoustic phonon scattering, the momentum relaxation rates diverge as $E_{K}$ approaches zero.

In Fig. 2 the scattering rates for scattering from alloydisorder is shown as a function of the parameter $2 K R$. For a given $R$, scattering rate increases with decreasing $2 K R$. From the figure, we can see that the scattering rate increases and the momentum relaxation time decreases as the radius of the wire decreases for scattering alloydisorder. It is shown that for alloy-disorder and impurity scattering the momentum relaxation rates diverge as $2 K R$ approaches zero.

In Fig. 3 the composition dependence of the electron mobility is shown for the $\mathrm{In}_{1-\mathrm{x}} \mathrm{Ga}_{\mathrm{x}}$ As quantum well wire with the radius $R=100 \mathrm{~A}^{0}$. Curves 2,3 in Fig. 2 show the composition dependence of the electron mobility for the $\mathrm{In}_{1-\mathrm{x}} \mathrm{Ga}_{\mathrm{x}}$ As quantum well [11] and bulk $\operatorname{In}_{1-\mathrm{x}} \mathrm{Ga}_{\mathrm{x}}$ As [10], respectively. In three cases bulk density of electrons is the same $n_{3 D}=2 \cdot 10^{17} \mathrm{~cm}^{-3}$. The strength of the alloy disorder potential $\mathrm{d} V$ is chosen to be $0.53 \mathrm{eV}$.

The nature of the composite dependence of the electron mobility limited by alloy disorder scattering is given by $\left[x(1-x) m *(x) a_{0}(x)^{3}\right]^{-1}$. Here $a_{0}(x)$ is the lattice parameter and $m^{*}(x)$ is the electron effective mass at the composition $x$.

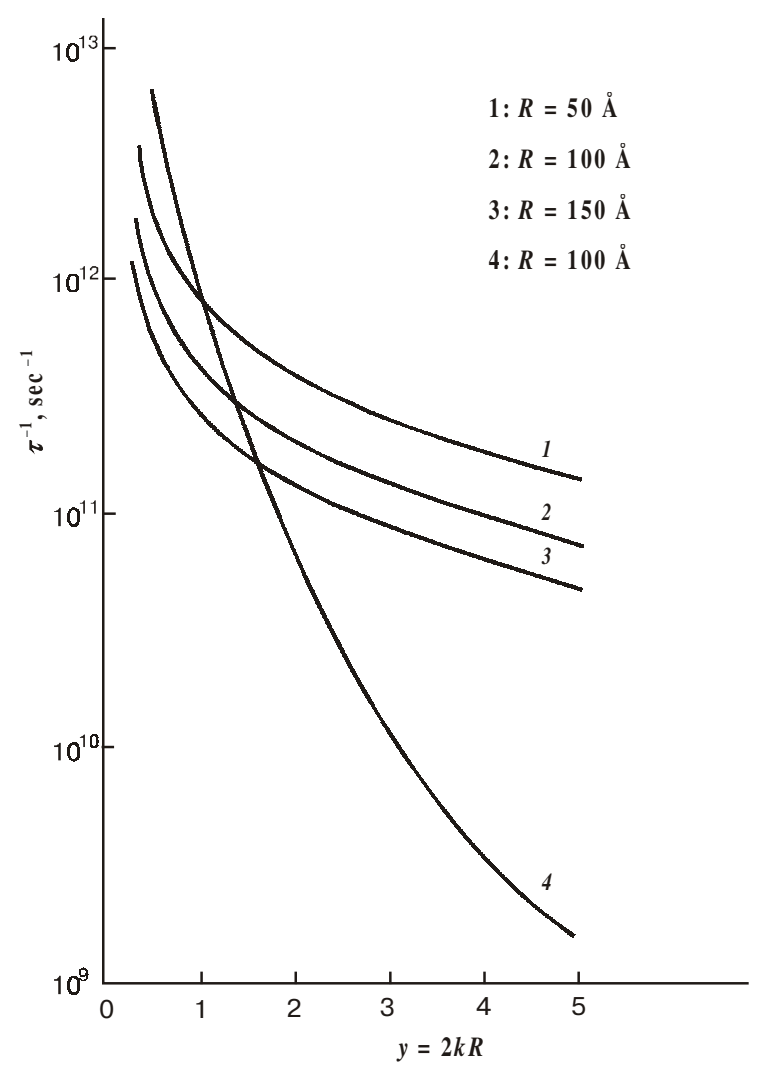

Fig. 2. Momentum relaxation rate is shown as a function of $2 K R$ for various well wire radii due to scattering caused by the alloydisorder (curve 1-3) and scattering caused by background impurities (curve 4 [25]).

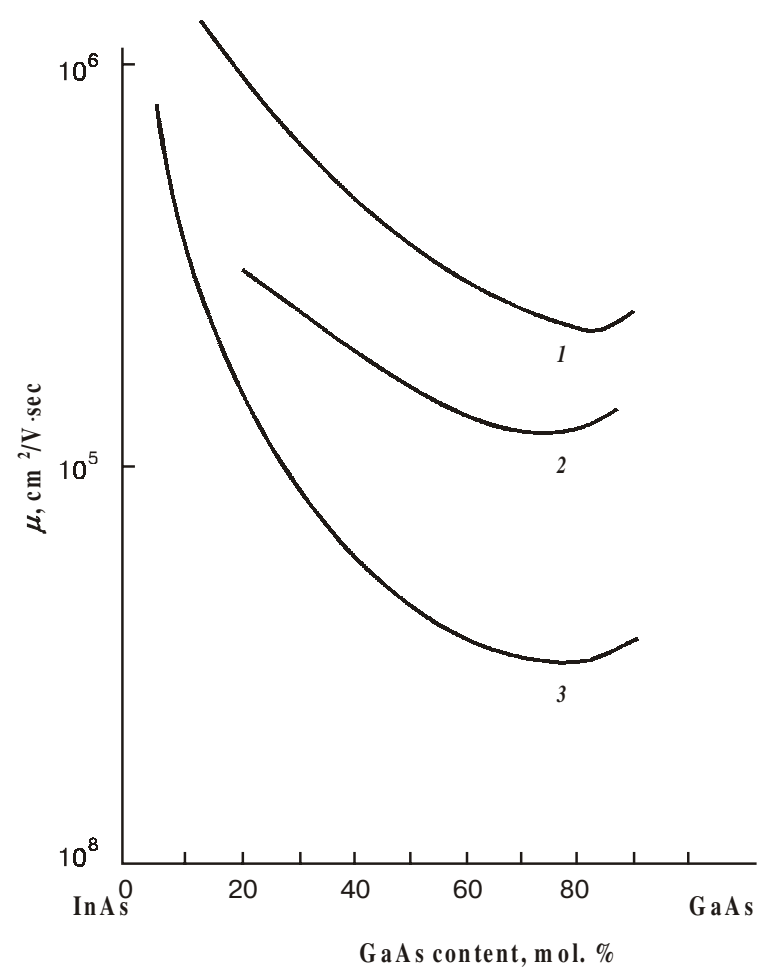

Fig. 3. Dependence of the alloy-disorder scattering limited electron mobility on the alloy composition for the case of $\mathrm{Ga}_{\mathrm{x}} \operatorname{In}_{1-\mathrm{x}} \mathrm{As}$ quantum wire (curve 1), quantum well structure (curve 2, [11], and 3D systems (curve 3, [10] ).

SQO, 5(4), 2002 


\section{G.B. Ibragimov: Alloy scattering in quantum well wire structures ...}

In Fig. 4 the variation of electron mobility limited only by the wire radius $R$. It can be seen that mobility $\mathrm{m}$ increases with increasing the wire radius. It is also shown that $\mathrm{m}$ decreases with decreasing temperatures.

In Fig. 5 the variation of electron mobility limited only by the alloy-disorder scattering is shown as a function of the electron density for $\mathrm{Ga}_{0.2} \mathrm{In}_{0.8} \mathrm{As}, \mathrm{Ga}_{0.3} \mathrm{In}_{0.7} \mathrm{As}$ and $\mathrm{Ga}_{0.47} \mathrm{In}_{0.53} \mathrm{As}$ cylindrical wires with the radius $R=$ $=100 \mathrm{~A}^{0}$. Thus the alloy-disorder scattering is important at low concentrations especially for systems with a small Ga content $x$.

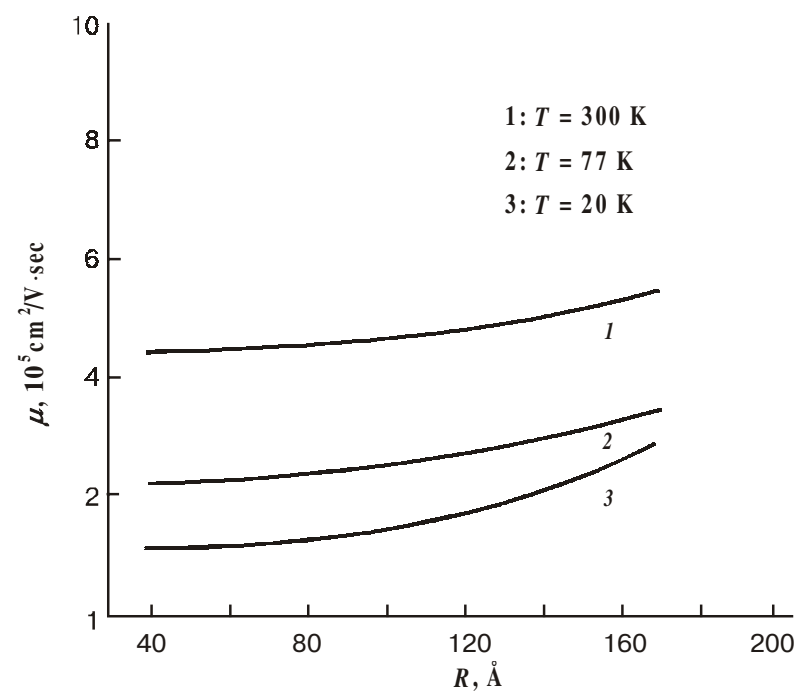

Fig. 4. Mobility for scattering from alloy-disorder as a function of wire radius $R$ in $\mathrm{Ga}_{0.47} \mathrm{In}_{0.53}$ As cylindrical wire for various temperatures.

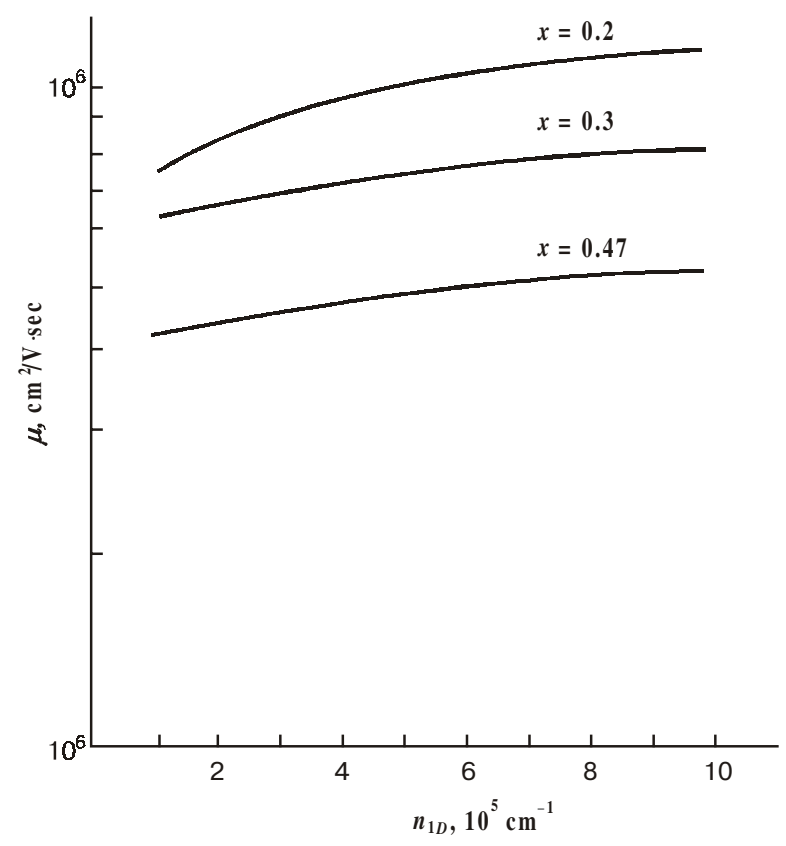

Fig. 5. Variation of the mobility versus the linear density of electrons for different alloy compositions.
In conclusion, we have developed the theory for mobility assuming scattering of electrons by alloy-disorder, and have found that this limiting value is higher than the mobility of the Q2D alloy. From our numerical results presented here, it has been shown that alloy-disorderscattering-limited mobility depends upon the wire radius and temperature.

\section{Acknowledgements}

The author would like to thank M.I. Aliev, B.M. Askerov and F.M. Gashimzade for helpful discussions.

\section{References}

1. H. Sakaki. Scattering Suppression and High-Mobility Effect of Size-Quantized Electrons in Ultrafine Semiconductor Wire Structures // Jpn.J.Appl.Phys. 19(12), pp. L735-1739 (1980).

2. J. Lee and H.N. Spector.Impurity-limited mobility of semiconducting thin wire // J.Appl.Phys. 54 (7), pp. 39213925 (1983).

3. G.Fishman. Phonon-limited mobility in a quasi-one-dimensional semiconductor // Phys.Rev.B. 36(14), pp. 7448-7455 (1987).

4. J. Lee and M.O. Vassel. Low-field electron transport in quasione-dimensional semiconducting structures // J.Phys.C: Solid State Phys. 17, pp. 2525-2535 (1984).

5. Wu and C.J. Lin. Impurity-limited mobility of semiconducting thin wires in n-type gallium arsenide // J.Appl.Phys. 83(3), pp. 1390-1395 (1988).

6. T. Ando, A.B. Fowlers, and F.T. Stern. Electronic properties of two-dimensional systems // Rev.Mod.Phys. 54, pp. 437672 (1982).

7. S. Briggs and J.P. Leburton // Phys.Rev.B. 38, p. 8163 (1988).

8. V.K. Arora. Size-Dependent Electric Conductivity in Semiconducting Thin Wires // Phys.Status Solidi B105, pp.707-713 (1981).

9. S. Kundu, C.K. Sakar and P.K. Basu. Low field mobility and thermopower in one-dimensional electron gas // J.Appl.Phys. 68(3), pp. 1070-1074 (1990).

10. L.Makowski and M.Gliksman. Disorder scattering in solid solutions of III-V semiconducting compounds. // J.Phys. Chem. Solids 34, pp.487-492 (1973).

11. S.B. Ogale and A. Madhukar.Alloy disorder scattering contribution to low-temperature electron mobility in semiconductor quantum well structures // J.Appl.Phys. 56(2), pp. 368374 (1984).

12. J.W. Harrison and J.R. Hauser.Theeoretical calculations of electron mobility in ternary III-V compounds // J.Appl.Phys. 47(1), 292-300 (1976).

13. P.K. Basu and K. Bhattachayya. Modification of the Theory of Two-Dimensional Mobility Due to Scattering by Alloy clusters Phys. Stat.Sol. (b), 128 pp.K175-177 (1985).

14. Ray and P.K. Basu.Alloy-disorder-scattering-limited mobility of electrons in superlattice // Phys.Rev. B46(16), pp. 9169-9172 (1992).

15. M.I. Aliev, Kh.A. Khalilov, G.B. Ibragimov. Free-carrier absorption in $\operatorname{In}_{1-\mathrm{x}} \mathrm{Ga}_{\mathrm{x}} \mathrm{As} / /$ Phys.Stat.Sol.(b), 140, pp. K8386 (1987).

16. G.B. Ibragimov, Free-carrier absorption in semiconducting quantum wells for alloy-disorder scattering // J.Phys.: Condens. Matter 14, pp. 4977-4983(2002).

17. J.Mycieski, G.Bastard and C. Rigaux. Intraband magnetoabsorption in mixed semiconductors with composition fluctuations and in imperfectt semiconductors // Phys. Rev. B16(4), 1675-1684 (1977). 


\section{G.B. Ibragimov: Alloy scattering in quantum well wire structures ...}

18. G. Bastard. Energy levels and alloy scattering in InPIn(Ga)As heterojunctions // Appl.Phys. Letters 43(6), pp.591593 (1983)

19. T. Ando. Self-Consistent Results for a GaAs/ $/ \mathrm{Al}_{\mathrm{x}} \mathrm{Ga}_{l_{-\mathrm{x}}} \mathrm{As}$ Heterojunction.II. Low Temperature Mobility // J.Phys.Soc.Jap. 51(12), pp. 3900-3907 (1982).

20. S. Jaziri and R. Ferreira. Excitonic states of weakly confining quantum wires // J.Appl.Phys. 84( 2), 893-900 (1998).

21. D.Chattopadhyay. Alloy scattering in quantum-well structures of semiconductor ternaries // Phys.Rev. B31(2), pp.11451146 (1985).

22. U.Bockelmann,G.Abstreiter, G.Weimann, and W.Schlapp. Single-particle and transport scattering times in narrow GaAs/Al $\mathrm{Aa}_{\mathrm{x}} \mathrm{As}$ quantum wells // Phys.Rev. B41(11), pp. 7864-7867 (1990).

23. Z. Ikonic, P. Harrison, and R.W. Kelsall. Intersubband holephonon and alloy disorder scattering in SiGe quantum wells // Phys.Rev. B64, pp. 245311 (2001).
24. G.B. Ibragimov. Electron mobility and electron scattering by alloy-disorder in quasi-one-dimensional structure.Int. Conf. Amorphous and Microcrystal. Semiconduct. (St.Petersburg2002).

25. J.W. Brown and H.N. Spector. Impurity scattering limited momentum relaxation time in a quantum well wire // J.Vac.Sci.Technol. B4(2) pp. 453-458, (1986).

26. Chhi-Chong $\mathrm{Wu}$ and Chay-Jy Lin, Impurity-limited mobility of semiconducting thin wires in n-type gallium arsenide // $J$. Appl. Phys. 83(3), pp.1390-1395 (1998).

27. B.M. Askerov. Electron Transport Phenomena in Semiconductors (Singapore: World Scientific) 1994.

28. A.Gold, A. Ghazali.Analatical results for semiconductor quantum-well wire: Plazmons, shallow impurity states, and mobility // Phys.Rev. B41(11), pp. 7626-7640,(1990). 